\title{
Propiedades psicométricas de la Escala de Satisfacción con la Vida en los Estudiantes (SLSS) de Huebner en niños y niñas de 10 a 12 años de Chile
}

\author{
Jaime Alfaro*, Javier Guzmán, David Sirlopú, Catalina García, Fernando Reyes y Lorraine Gaudlitz
}

Universidad del Desarrollo. Facultad de Psicología (Chile).

\begin{abstract}
Resumen: Este trabajo analiza las propiedades psicométricas de la escala SLSS de Huebner en una muestra de niños y niñas chilenos. Se aplicó a 1096 estudiantes de 10 a 12 años $(M=11 ; D T=0.89)$ que asistían a establecimientos municipales, particulares subvencionados y particulares pagados en las ciudades de Santiago, Valparaíso y Concepción. Se realizaron análisis de fiabilidad de consistencia interna, análisis factorial exploratorio $(A F E)$ y confirmatorio $(A F C)$. Los resultados exhiben una aceptable fiabilidad en tanto consistencia interna de la escala $(\alpha=.86)$, el análisis factorial arroja dos factores en la estructura factorial de la escala que se corresponden con satisfacción con la vida y deseo de cambio. Se discute la solución bifactorial del modelo seleccionado en tanto no se corresponde con lo comunmente reportado en otras investigaciones. El estudio reporta resultados del uso de SLSS en población infantil y aporta al uso y desarrollo de herramientas de medición de autorreporte para el estudio del bienestar subjetivo en niños chilenos.

Palabras clave: propiedades psicométricas; bienestar subjetivo;
\end{abstract} satisfacción con la vida; SLSS, infancia.
Title: Psychometric properties of Huebner's Satisfaction with Life in Students Scale (SLSS) in Chilean boys and girls between 10 and 12 years old. Abstract: This article analyzes the psychometric properties of the Huebner's SLSS in a sample of Chilean boys and girls. 1096 students participated in the study who had between 10 and 12 years old $(M=11$; $S D=0.89$ ) and attended public, semiprivate, and private schools in Santiago, Valparaíso and Concepción. The analysis includes reliability analysis of internal consistency, exploratory factor analysis $(E F A)$, and confirmatory factor analysis $(C F A)$. Results show an acceptable reliability of the scale $(\alpha=.86)$, and factor analysis indicates two factors in the factor structure of the scale that correspond to life satisfaction and desire of change. The discussion examines the bi-factor solution of the model because this finding does not fit the conclusions of previous research. This study expands the utilization of the SLSS in child population contributing to the development of assessment tools for social indicators that consider the self-report of subjective well-being in Chilean children.

Key words: psychometric properties; subjective well-being; satisfaction with life; SLSS, childhood.

\section{Introducción}

\section{Bienestar Subjetivo y Satisfacción Global con la Vida}

La investigación en calidad de vida ha sido conceptualizada desde dos perspectivas: objetivas y subjetivas. El punto de vista objetivo se centra en las condiciones externas que contribuyen a la calidad de vida, tales como los niveles de ingresos, calidad de la vivienda, redes de amistad, y el acceso a los servicios de salud. En cambio la perspectiva subjetiva refiere a los juicios internos de los individuos con respecto a sus condiciones de vida. Estos juicios refieren a Satisfacción con la Vida, en general y/o ámbitos específicos de la vida (v.g., satisfacción con amigos, con experiencias escolares, con la familia, etc.). De tal manera que, si bien las circunstancias objetivas de vida son importantes en la determinación de la calidad de vida de los individuos, las interpretaciones y evaluaciones subjetivas de sus experiencias son más cruciales (Valois, Zullig, Huebner, \& Drane, 2004a).

La noción de Bienestar Subjetivo está estrechamente ligado a la satisfacción con diferentes ámbitos de la vida, y con una evaluación más integral, denominada Satisfacción Global con la Vida (Figuer et al., 2004). Para Campbell, Converse y Rodgers (1976) estos procesos se pueden medir a través de preguntas de satisfacción, de forma tal que, según Cummins, Eckersley, Van Pallant, Van Vugt, y Misajon (2003), la esencia del bienestar subjetivo está referida de mejor forma a las evaluaciones que hace una persona sobre la satisfacción

* Dirección para correspondencia [Correspondence address]: Jaime Alfaro. Facultad de Psicología, Universidad del Desarrollo. Av. La Plaza N680, Las Condes, Santiago de Chile (Chile).

E-mail: jalfaro@udd.cl con su vida. Diener (2006) considera que el bienestar subjetivo refiere a varios tipos de opiniones, buenas o malas, que la gente tiene sobre sus vidas, incluyendo evaluaciones cognitivas, como la satisfacción en el trabajo y la vida, el interés y el compromiso, así como las reacciones afectivas frente a acontecimientos de la vida como alegrías y tristezas. Petito y Cummins (2000) entienden el bienestar en términos de la evaluación individual que una persona hace de su calidad de vida, la cual está formada por dos componentes: el primero es la satisfacción de vida, lo que implica una evaluación cognitiva, y el segundo es emocional, e implica sentimientos y estado de ánimo.

Según Diener (1984), hay un fuerte consenso sobre tres características básicas del bienestar subjetivo: (1) se construye a partir de las experiencias de cada persona y de la percepción y evaluación de tales experiencias, (2) incluye dimensiones positivas y no solamente la ausencia de negativas y (3) incluye algunas evaluaciones globales de la vida. Delimitando aún más esta noción, se entiende que el bienestar subjetivo está constituido por tres dimensiones interrelacionadas: los afectos positivos, los afectos negativos y la satisfacción con la vida (Diener \& Suh, 1997; Pavot \& Diener, 1993). Otros autores consideran también el componente felicidad (Casas, 2010a).

En este marco, específicamente la satisfacción con la vida es entendida como una evaluación global de la persona sobre la calidad de su vida ante sus circunstancias (Seligson, Huebner, \& Valois, 2003) que da cuenta del grado en que una persona evalúa positivamente su vida como globalidad (Veenhoven, 1994), o con referencia a dominios específicos, como la familia, amigos, o la escuela (Huebner, 2004; Seligson, Huebner, \& Valois, 2005), relativa a un estado presente (Veenhoven, 1994). La relevancia de medir la 
satisfacción con la vida dice relación con que ella permiten calcular la calidad de vida "realizada" en un país o categoría social, generando datos útiles en la validación empírica de las condiciones necesarias para el vivir bien, y con ello informar acerca de qué es una buena sociedad (Veenhoven, 1994). Además, permite evaluar los efectos en la calidad de vida de las políticas aplicadas, siendo de esta manera de gran importancia para trazar estrategias de desarrollo social (Veenhoven, 2002). De este modo, el estudio y desarrollo de medidas de Satisfacción con la Vida proporciona una importante información para la prevención, detección precoz y la intervención en diversas poblaciones en condiciones de riesgo (Seligson et al., 2005).

\section{Bienestar Subjetivo en la infancia}

La disponibilidad de estudios sobre el bienestar de niños, niñas y adolescentes dista mucho de alcanzar la cantidad de resultados que ofrece la investigación con adultos (Casas, 2010a; Casas et al., 2012; Gadermann, Schonert-Reichl, \& Zumbo, 2010; Huebner \& Gilman, 2002; Huebner, 2004; Seligson et al., 2003; Tomyn \& Cummins, 2011) tanto cuantitativa como cualitativamente (Casas, 2010a). A la vez los pocos datos disponibles sugieren que durante las etapas más jóvenes de la vida el funcionamiento del bienestar tiene su propia idiosincrasia evolutiva a lo largo de los años, diferenciándose por tanto según edad (Casas, 2010a).

De igual forma, el estudio del bienestar subjetivo en la infancia y adolescencia es un campo que requiere desarrollo y avance investigativo en cuanto gran parte de los trabajos producidos refieren de manera principal a población en condiciones médicas crónicas, concentrándose en el campo de la pediatría, principalmente la investigación de la calidad de vida en niños con enfermedades crónicas (Casas, 2010b; Pollard \& Lee, 2003)

También se requiere avances en el desarrollo de instrumental para la medición del bienestar subjetivo en la infancia y adolescencia, tanto para aumentar la comprensión de los correlatos de éste, como para medir su estado y de igual forma guiar acciones tendientes a su elevación y mejora, complementariamente a una aproximación centrada en la evaluación de las conductas problemáticas o dimensiones patológicas de éstas (Gadermann, et al., 2010). Además, contar con este intrumental permitiría conocer los factores relacionados con las diferencias individuales en la percepción del bienestar y sus secuelas (Huebner \& Gilman, 2002; Seligson et al., 2003, 2005), así como la identificación de subgrupos de riesgo para garantizar la adecuada prestación de apoyo y recursos a los que lo necesitan (Tomyn \& Cummins, 2011).

En particular se requiere desarrollar medidas comparativas nacionales y transnacionales, y de grupos o entornos culturales diversos (Casas, 2010a; Casas et al., 2012; Siyez \& Kaya, 2008), ya que existen hasta ahora escasas publicaciones con prueba empírica de los resultados obtenidos con diferentes instrumentos aplicados a infancia y adolescencia en diferentes países o contextos culturales, siendo la mayoría de éstos realizados en el mundo anglosajón, con algunas excepciones (Casas et al., 2012).

La investigación en bienestar subjetivo en población infantil y adolescente (y también adulta), no se ha producido de manera significativa en países en vías de desarrollo, tal cual reportan diversos autores (Lau, Cummins, \& McPherson, 2005; Tiliouine, Cummins, \& Davern, 2006; Siyez \& Kaya, 2008; Webb, 2009; Yiengprugsawan, Seubsman, Khamman, \& Lim, 2010). Entre los pocos trabajos realizados en países en vías de desarrollo se conocen por ejemplo reportes del Índice de Bienestar Personal (PWI) aplicados en países, tales como Tailandia (Yiengprugsawan et al., 2010), Argelia (Tiliouine et al., 2006), o con la Escala Breve Multidimensional de Satisfacción con la Vida en Estudiantes (BMSLSS) en Turquía (Siyez \& Kaya, 2008). Uno de los avances en América Latina, fue realizado en 2010 mediante el estudio transcultural sobre bienestar subjetivo en Chile, Brasil, Argentina y España en adolescentes de 12 a 16 años (Casas et al., 2011).

Es necesario avanzar en América Latina de manera sistemática en el conocimiento sobre calidad de vida de los niños y adolescentes, y en particular sobre su calidad de vida en dimensiones subjetivas, según sus diferentes contextos y la diversidad de situaciones colectivas de la región (Aguado, 2009). Es así que según investigadores internacionales del campo (Ben-Arieh, 2008; Bradshaw \& Richarson, 2009; Bradshaw \& Lau, 2010; Casas, 2010b) se requiere específicamente desarrollar indicadores sobre Infancia que: Contemplen el uso de indicadores de resultado y mediciones directas del bienestar más que el uso de indicadores indirectos; tengan como unidad de análisis y de información al niño (en vez de los padres, la familia o el hogar); den prioridad a indicadores del bienestar actual de los niños, más que a indicadores de futuro bienestar o realización como adultos (diagnosticar qué ocurre con sus condiciones de vida en el presente); usen indicadores sobre sus sentimientos y su vida, lo que se suele denominar indicadores subjetivos.

En Chile, se han validado escalas en adolescentes, y estudiado aspectos que predominan en la visión que los adolescentes tienen sobre su satisfacción global con la vida (Alfaro, Valdenegro, \& Oyarzún, 2013) y además se ha analizado psicométricamente instrumental sobre bienestar psicológico, como la escala Ryff (versión española) en población adolescente (Gallardo \& Moyano, 2012). En adultos mayores, se ha adaptado y validado instrumentos como la escala de felicidad de MUNSH (Moyano, Flores, \& Soromaa, 2011). Adicionalmente, se ha estudiado el bienestar subjetivo utilizando la escala de Satisfacción con la Vida de Diener, analizando sus propiedades psicométricas en una aplicación en adultos entre 18 y 65 años (Vera-Villarroel, Urzúa, Pavez, Celis-Atenas, \& Silva, 2012). Pese a estos sustantivos aportes, no se cuenta con estudios de bienestar en población infantil de 10 a 12 años, así como tampoco con desarrollo de instrumental válido para este grupo de edad. 


\section{Satisfacción con la Vida y problemas sociales}

Gran parte de la relevancia de avanzar en este campo dice relación con que el bienestar subjetivo, en sus distintas acepciones, se ha relacionado con la integración social de las personas, la salud y la organización comunitaria. Así como también el acceso a medidas de satisfacción global con la vida, a la vez que por ámbitos de satisfacción, resulta ser útil con niños, niñas y adolescentes, pues en combinación con escalas tradicionales orientadas a la patología, predice importantes resultados de salud mental (Huebner, Suldo, \& Valois, 2003). Específicamente el estudio de la satisfacción con la vida permite determinar riesgos en dimensiones tanto psicológicas como sociales, tiene una valiosa capacidad predictiva respecto de estados patológicos, así como en la capacidad de manejo de eventos vitales estresantes y sus efectos en problemas de comportamiento (Puente \& Cavazos, 2013). Contribuye de esta manera con información clave para intervenciones socio-sanitarias como la prevención y detección precoz, en diversas poblaciones en condiciones de riesgo.

Las medidas de satisfacción o insatisfacción vital son útiles también para propósitos clínicos, en tanto resultados por debajo del punto neutro en las escalas (insatisfacción) proporcionan información relevante del estado de bienestar de niños que no presentan indicios de psicopatología. Mediciones tradicionales de evaluación psicológica parecen dar a entender que los niños que no presentan psicopatología tendrían necesariamente un adecuado nivel de bienestar subjetivo, lo que según la evidencia pareciera ser engañoso, en cuanto los niños que no presentan signos de patología clínicamente relevante (por ejemplo, síntomas depresivos) no necesariamente experimentan niveles adecuados de bienestar subjetivo. Como resultado, algunos grupos de niños pueden pasar desapercibidos por los profesionales de salud mental, dificultando la implementación de medidas preventivas tempranas (Seligson et al., 2005).

Se cuenta con evidencia respecto de su utilidad predictiva, en relación a que la medición de la Satisfacción con la Vida ha demostrado asociación con conductas de riesgo en los jóvenes (Paxton, Valois, Huebner, \& Drane, 2006). Específicamente, mediciones de adolescentes con la BMSLSS han demostrado utilidad predictiva en relaciones simultáneas, negativas con el alcohol, el tabaco y el consumo de drogas ilegales, así como con comportamientos de riesgo, tales como conducta agresiva y el uso de armas (Huebner et al., 2003).

La evaluación de la satisfacción vital puede ser útil también en cuanto los estudios con adolescentes muestran asociaciones significativas entre esta dimensión y comportamientos tales como, la asunción de riesgos sexuales, ideación y comportamiento suicida (Valois et al., 2004a), problemas adaptativos relacionados con la alimentación (Valois, Zullig, Huebner, \& Drane, 2003), y el ejercicio y la actividad física (Valois, Zullig, Huebner, \& Drane, 2004b). Otra investigación con adolescentes indica que la satisfacción con la vida en general desempeña un papel mediador en la relación entre los eventos estresantes de la vida y conductas internalizantes en adolescentes (McKnight, Huebner, \& Suldo, 2002), y un papel moderador de la relación entre los eventos estresantes y conductas externalizantes en adolescentes (Huebner, Suldo, Valois, \& Drane, 2006).

En términos generales, la satisfacción con la vida está integralmente vinculada a una amplia gama de indicadores físicos, mentales, de rendimiento escolar, emocionales y sociales de funcionamiento humano, lo que sugiere que esta noción da cuenta de una fortaleza psicológica clave (Jiang, Huebner, \& Hills, 2013), por lo que las medidas de bienestar subjetivo y satisfacción con la vida son una herramienta relevante que permite extender el alcance de los indicadores de bienestar más allá de la primacía de los indicadores objetivos comunmente utilizados en la toma de decisiones gubernamentales (Valois et al., 2003). Por último, también es relevante tener en cuenta que en la satisfacción con la vida, según recogemos de Athay, Kelley y Dew-Reeves (2012), juegan un rol relevante los mecanismos internos (como por ejemplo, las percepciones) presentando importantes influencias en la satisfacción de la vida, con más peso que las condiciones objetivas, o las variables demográficas.

\section{Escala de Satisfacción Vital de los Estudiantes (SLSS)}

Esta escala fue creada en 1991 por Huebner con el fin de medir la satisfacción con la vida en forma global de niños y adolescentes entre 8-18 años de edad. La SLSS consta de 7 ítems con 6 opciones de respuesta tipo Likert, donde $1=$ totalmente en desacuerdo y $6=$ totalmente de acuerdo (Weber, Ruch, \& Huebner, 2013). Mediante la suma de los puntajes de cada respuesta se obtiene un índice global de satisfacción vital. Los puntajes de la escala van de 7 a 42, donde un mayor puntaje significa mayor satisfacción con la vida en general (Marques, Pais-Ribeiro, \& Lopez, 2007).

En los resultados de la aplicación de la SLSS en población adolescente de diversos países y edad se presenta como un instrumento fiable. Los coeficientes de consistencia interna Alfa de Cronbach reportados oscilan entre $\alpha=.79$ a $\alpha=$ 89, tanto en población clínica como no clínica (Chaves, Vazquez, \& Hervas, 2013; Galindez \& Casas, 2010; Huebner, 1991; Marques et al., 2007; Weber et al., 2013). Los siete ítems conforman un solo factor de satisfacción vital que explica alrededor del 50\% de la varianza total (Galindez \& Casas, 2010).

Al ser aplicada en modalidad test-retest los coeficientes obtenidos son $r=.74$ en intervalos de 1 a 2 semanas (Huebner, 1991) y $r=.55$ en intervalos de cuatro meses (Weber et al., 2013) lo que indica que la escala SLSS mantiene significativa estabilidad temporal. El apoyo para la validez de constructo de esta escala lo proveen las relaciones entre la SLSS y otras medidas de bienestar, como la BMSLSS ( $r=$ .70), la Escala de Autoconcepto de Piers-Harris $(r=.53)$ y su 
subescala de felicidad $(r=.53)$, la Escala de Satisfacción Vital de Andrews y Withney $(r=.62)$, la Self-Anchoring Striving Scale (SASS) $(r=.61)$ y con Fordyce Happiness Measure (FHM) $(r=$ .53) (Galindez \& Casas, 2010; Huebner, 1991; Seligson et al., 2003; Weber et al., 2013).

En cuanto a la validez concurrente con población adolescente, se han encontrado relaciones fuertemente significativas con autoestima $(r=.57)$ y con autoconcepto $(r=.60)$. Por el contrario, presenta una correlación inversa con ansiedad $(r=-.52)$, (Galindez \& Casas, 2010). Existe una correlación moderada con deseabilidad social (Huebner, 1991; Weber et al., 2013) y correlaciones no significativas con nivel educacional y coeficiente intelectual (Marques et al., 2007).

Los resultados de la SLSS en adolescentes indican que la escala no está influenciada por diferencias sociodemográficas; no se ha encontrado efecto significativo del género ni diferencias significativas entre hombres y mujeres. Se observa una leve y moderada disminución de los puntajes a medida que la edad aumenta (Huebner, 1991; Marques et al., 2007; Weber et al., 2013). En este contexto, el presente trabajo tiene como objetivo estimar las propiedades psicométricas de la Escala de Satisfacción Vital de los Estudiantes (SLSS) de Huebner (1991) en una muestra de niños y niñas chilenos de 10 a 12 años. Adicionalmente, se examinará la validez convergente de esta escala con la Escala Multidimensional Breve de Satisfacción con la Vida para Estudiantes (BMSLSS).

\section{Método}

\section{Diseño}

Debido a que el propósito es presentar las propiedades psicométricas de una escala, el diseño de investigación empleado es de tipo instrumental (Ato, López, \& Benavente, 2013; Montero, \& León, 2007).

\section{Participantes}

La muestra empleada corresponde a un muestreo aleatorio por conglomerados polietápico, seleccionado primariamente establecimientos educacionales en tres ciudades considerando los tres tipos de administración educacional existentes en el sistema escolar chileno (Municipal, Particular subvencionado y Particular pagado), posteriormente se seleccionaron cursos y de éstos se seleccionaron los estudiantes. La muestra finalmente quedó conformada por 1096 niños y niñas (498 mujeres; 598 hombres), con edades entre 10 y 12 años $(M=11$ años, $D T=.89)$, pertenecientes a tres ciudades de Chile: Santiago (27\%), Valparaíso (34.2\%) y Concepción $(38.8 \%)$. En las tres ciudades la selección consideró la proporcionalidad de los tipos de administración existentes en el sistema escolar chileno. De esta forma la distribución de dependencia escolar de los participantes fue la siguiente: establecimientos municipales $(57.8 \%)$, particular subvencionados $(35.3 \%)$ y particulares pagados $(6.9 \%)$.

\section{Instrumentos}

Escala de Satisfacción Vital de los Estudiantes (SLSS)

En esta investigación se empleó la versión en español de la SLSS (Huebner, 1991) que fue traducida por los autores de este estudio siguiendo las indicaciones de la International Test Commission, evaluándose específicamente los criterios referidos al formato del ítem, aspectos de gramática y redacción, así como aspectos culturales (Muñiz, Elousa, \& Hambleton, 2013). De esta forma se realizó traducción y retro-traducción por profesionales calificados en idioma inglés, además de una aplicación piloto a escolares equivalentes en rango etario y tipo de establecimiento educacional de la muestra final. Como resultado de dicha aplicación se hicieron algunos ajustes idiomáticos y de formato de la escala teniendo en consideración las características de la población estudiada. La SLSS contiene siete ítems que evalúan aspectos globales de la vida de los escolares. En la Tabla 1 aparecen los ítems originales en inglés y la traducción que se utilizó en esta investigación. La escala de respuesta utilizada fue tipo Likert de 5 niveles ( 0 $=$ Totalmente en desacuerdo $; 4=$ Totalmente de acuerdo).

Tabla 1. Traducción al Español de los ítems de la SLSS

\begin{tabular}{ll}
\hline Original & Traducción \\
\hline 1. I have a good life & Tengo una buena vida \\
2. I have what I want in life & Tengo lo que quiero en la vida \\
3. My life is better than most & Mi vida es mejor que la de otros \\
kids & niños y niñas \\
4. My life is going well & Mi vida va bien \\
5. My life is just right & Mi vida es como yo quiero que \\
& sea \\
6. I would like to change many & No) Me gustaría cambiar mu- \\
things in my life & chas cosas en mi vida \\
7. I wish I had a different kind & No) Me gustaría tener otro tipo \\
of life & de vida \\
\hline
\end{tabular}

Escala Multidimensional Breve de Satisfacción con la Vida para Estudiantes (BMSLSS)

Escala creada por Seligson et al. (2003), en esta investigación se empleó la versión en español traducida por los autores la que fue sometida a procedimiento de traducción-retrotraducción, además de aplicarla a una muestra piloto de 290 estudiantes de dos ciudades donde se llevó a cabo el estudio, como resultado de esta aplicación se hicieron algunos ajustes idiomáticos (Alfaro et al., 2015). La BMSLSS está formada por 6 preguntas cerradas de auto-reporte, que indagan sobre distintos dominios importantes en la vida de los niños. La escala de respuesta empleada fue de tipo Likert de 11 niveles $(0=$ Totalmente descontento $/ a ; 10=$ Totalmente contento $/ a)$. Las 6 preguntas son: (1) ¿Hasta qué punto te encuentras satisfecho o satisfecha con tu vida familiar?; (2) ¿Hasta qué punto te encuentras satisfecho o satisfecha con tus amigos y amigas?; (3) ¿Hasta qué punto te encuentras satisfecho satisfecha con el barrio donde vives en general?; (4) ¿Hasta qué punto te encuentras satisfecho o satisfecha con tu experiencia en el colegio o liceo?; (5) ¿Hasta qué punto te encuentras satisfe- 
cho o satisfecha contigo mismo/a?; (6) ¿Hasta qué punto te encuentras satisfecho o satisfecha con toda tu vida en general?. El Alfa de Cronbach para esta escala fue de .70. Los ítems fueron promediados para obtener el puntaje total de la BMSLSS, que representa la satisfacción general con la vida.

\section{Procedimiento}

Los directores de los colegios y los centros de padres de los establecimientos educacionales fueron contactados telefónicamente para contarles el objetivo general del estudio e invitarlos a participar. Las instituciones que accedieron a colaborar, debieron firmar un consentimiento institucional para que sus alumnos pudieran participar. Posteriormente, todos los escolares fueron invitados a participar de modo voluntario en el estudio; los que respondieron afirmativamente debieron firmar un asentimiento. El cuestionario se aplicó a través de la modalidad de autoadministración y en horarios de clases, con previa autorización del profesor a cargo, durante el período escolar 2009 y 2010. Los encuestadores que realizaron esta tarea fueron en su mayoría estudiantes universitarios, inscritos en áreas de las ciencias sociales y/o educativas y con experiencia previa en levantamiento de datos. Se cumplieron los protocolos éticos de investigación que orientan la investigación científica de Chile (CONICYT/ FONDECYT, 2008), con especial énfasis en la confidencialidad de la información producida.

\section{Análisis de datos}

Para evaluar la estructura factorial de la escala se usó una estrategia de validación cruzada, para lo cual se dividió aleatoriamente la muestra en dos submuestras de igual tamaño: (a) la muestra de entrenamiento $(n=548)$ y (b) la muestra de validación $(n=548)$. La muestra de entrenamiento se empleó para llevar a cabo un análisis factorial exploratorio con la finalidad de identificar la estructura factorial de la escala. Como estrategia analítica se usó una rotación Geomin y el método Weigthed Least Square con ajuste a la media y la varianza (WLSMV). En estudios simulados realizados en ordenador, se ha observado que el WLSMV tiene un buen desempeño en general cuando se analizan datos ordinales (Beauducel \& Herzberg, 2006; Lei, 2009; Muthén, du Toit, \& Spisic, 1997), excepto cuando se emplean tamaños de muestra igual o menor a 200 casos (Kline, 2011).

Dado que los ítems de la escala se contestan empleando cinco opciones de respuesta de tipo ordinal, el supuesto de distribución normal multivariado no se cumple para estos datos, por lo que al momento de estimar los modelos AFE y AFC, se procedió a obtener la matriz de correlaciones policóricas y a continuación se llevó a cabo la estimación de los parámetros del modelo.

La calidad del ajuste de los modelos se llevó a cabo sobre la base de los estadísticos que usualmente son reportados en la literatura: (a) $\chi^{2}$, (b) CFI, (c) TLI, (d) SRMR, (e) RMSEA con un intervalo de confianza del $90 \%$ y (f) WRMR. Como criterios indicativos de un buen ajuste de un modelo se ha propuesto un $\chi^{2}$ no significativo, valores de 0.95 o superiores para el CFI y el TLI, valores inferiores a 0.10 para el RMSEA y un valor menor a 1.0 para el WRMR (Hu \& Bentler, 1999; Kline, 2011; West, Taylor, \& Wu, 2012).

Se llevó a cabo imputación de valores perdidos usando el algoritmo EM (Expectation-Maximization). El EM es usado típicamente para computar estimaciones de máxima probabilidad cuando existen datos perdidos (Cuesta, FonsecaPedrero, Vallejo, \& Muñiz, 2013). Los análisis de alfa ordinal y matrices policóricas se llevaron a cabo empleando el programa FACTOR 9.30 (Lorenzo-Seba \& Ferrando, 2006) y el $A F E$ y $A F C$ se llevó a cabo usando el programa Mplus 7.2 (Muthén \& Muthén, 2012).

\section{Resultados}

\section{Estadísticos descriptivos}

En primer término se analizó la forma de distribución de los ítems. Algunos ítems presentaron valores de asimetría y curtosis superiores a 1 (ver Tabla 2). La aplicación del coeficiente de normalidad multivariante de Mardia (1970, 1974) para estos indicadores muestra un valor de 16.93 para la asimetría $(p>.05)$ y de $88.43(p<.01)$ para la curtosis. Siguiendo a Ullman (2006), coeficientes mayores a 3.0 indican presencia de no normalidad. En la Tabla 2 también se consignan los promedios y desviación típica para cada ítem. Los ítems "mi vida va bien", "mi vida es como yo quiero que sea", "tengo una buena vida", "tengo lo que quiero en la vida", "mi vida es mejor que la de otros niños y niñas" y "me gustaría tener otra vida" tuvieron puntajes por encima del punto medio de la escala que, en este caso, fue 2. Por otra parte, los participantes mostraron un bajo grado de acuerdo con el ítem "me gustaría cambiar muchas cosas en mi vida" (invertido). 
Tabla 2. Medias Desviación Típica, Asimetría, Curtosis y Correlaciones Policóricas para los Ítems de la SLSS $(N=1096)$.

\begin{tabular}{|c|c|c|c|c|c|c|c|c|c|c|c|}
\hline Ítems & $M$ & $D T$ & $A$ & $C$ & 1 & 2 & 3 & 4 & 5 & 6 & 7 \\
\hline 1. Tengo una buena vida & 3.39 & .94 & -1.75 & 2.69 & - & & & & & & \\
\hline 2. Tengo lo que quiero en la vida & 3.18 & 1.01 & -1.18 & .81 & .68 & - & & & & & \\
\hline 3. Mi vida es mejor que la de otros niños y niñas & 2.53 & 1.55 & -.45 & -.45 & .45 & .51 & - & & & & \\
\hline 4. Mi vida va bien & 3.35 & .87 & -1.61 & 2.54 & .73 & .63 & .38 & - & & & \\
\hline 5. Mi vida es como yo quiero que sea & 2.90 & 1.42 & -.95 & .10 & .63 & .61 & .40 & .68 & - & & \\
\hline 6. Me gustaría cambiar muchas cosas en mi vida (i) & 1.60 & 1.48 & .45 & -1.20 & -.18 & -.13 & .01 & -.12 & -.12 & - & \\
\hline 7. Me gustaría tener otro tipo de vida (i) & 2.37 & 1.55 & -.35 & -1.40 & -.35 & -.22 & -.01 & -.25 & -.20 & .60 & - \\
\hline
\end{tabular}
Nota. $M=$ Media $D T=$ Desviación Típica; $A=$ Asimetría; $C=$ Curtosis; i = ítem inverso.

Puesto que los ítems de la SLSS fueron respondidos con un formato tipo Likert, por tanto, no son variables continuas y tampoco se distribuyen de forma normal, se decidió analizar los datos en base a una matriz de correlaciones policóricas, según lo recomendado en la literatura (Domínguez, 2014; Elosua \& Zumbo, 2008; Ferrando \& Lorenzo-Seva, 1994, 2014; Freiberg, Stover, de la Iglesia, \& Fernández, 2013; Llorent-Segura, Ferreres-Traver, Hernández-Baeza, \& Tomás-Marco, 2014). En la Tabla 2 aparecen las correlaciones policóricas entre los ítems de la SLSS para la muestra total. Según Olson (1979), cuando se utiliza este tipo de correlaciones, lo que se hace es evaluar la adecuación de la matriz de correlaciones a través de dos pruebas: el estadístico de Bartlett y la prueba de Kaiser-Meyer-Olkin (KMO). En este caso, el test de Bartlett fue igual a $2173.9(g l=21, p<.001)$ y el $K M O$ igual a 0.79 , lo que nos informa que la matriz es adecuada. En general, los cinco ítems que no fueron invertidos obtuvieron correlaciones altas entre sí y en la dirección teóricamente esperada. Sin embargo, estos ítems obtuvieron correlaciones inversas y bajas con los dos ítems invertidos ("me gustaría cambiar muchas cosas en mi vida" y "me gustaría tener otro tipo de vida"), pero una alta correlación entre ambos.

\section{Fiabilidad}

La estimación de la fiabilidad de las puntuaciones se realizó mediante el coeficiente alfa ordinal (Elosua \& Zumbo, 2008; Domínguez, 2012), de esta forma no se infraestima los resultados cuando se emplea una matriz policórica (Gadermann, Guhn, \& Zumbo, 2012), obteniéndose un alfa de .86 para la escala total.

\section{Validez convergente}

Para evaluar la validez convergente, se realizó una correlación bivariada de Pearson entre los puntajes de la SLSS y los de la BSMLSS. Se obtuvo una asociación positiva y moderada entre estas medidas $(r=.54, p<.001)$, lo cual apoya la validez convergente de la SLSS.

\section{Validez de constructo}

Para el AFE se utilizó el estimador Weigthed Least Square con ajuste en la media y la varianza (WLSMV) que es el más recomendable para trabajar con datos ordinales (Beauducel \& Herzberg, 2006; Lei, 2009; Muthén et al, 1997). En la Tabla 3 se presentan las cargas factoriales de los siete ítems. Como se puede apreciar, los dos últimos ítems invertidos obtuvieron cargas menores en comparación con el resto de ítems que cargaron en este factor. Los valores de los índices de ajuste de este $A F E$ fueron: $\chi^{2}(14)=843.86, p<.001$; RMSE $A=.34$ (90\% IC: .32-.36); CFI = .76; TLI= .64; $S R M R=.13$. Estos valores son bajos, lo que nos indica que en la estructura factorial de la SLSS pudiese existir otras dimensiones, por lo que se exploró analizar una solución con dos dimensiones.

Tabla 3. Cargas factoriales de los ítems del SLSS para las soluciones de un solo factor y dos factores.

\begin{tabular}{|c|c|c|c|c|}
\hline \multirow[b]{2}{*}{ Ítems } & \multirow{2}{*}{$\begin{array}{c}\text { Cargas factoriales del } \\
\text { AFE } \\
F 1\end{array}$} & \multicolumn{2}{|c|}{$\begin{array}{c}\text { Cargas factoriales del } A F C \text { para el } \\
\text { modelo de } 2 \text { factores }\end{array}$} & \multirow{2}{*}{$\begin{array}{l}\text { Cargas factoriales del } A F C \\
\text { para el modelo de } 1 \text { factor } \\
F 1\end{array}$} \\
\hline & & $F 1$ & F2 & \\
\hline Tengo una buena vida & $.88^{*}$ & $.87 *$ & & $.86^{*}$ \\
\hline Tengo lo que quiero en la vida & $.78^{*}$ & $.78^{*}$ & & $.78^{*}$ \\
\hline Mi vida es mejor que la de otros niños y niñas & $.47^{*}$ & $.48^{*}$ & & $.50 *$ \\
\hline Mi vida va bien & $.88^{*}$ & $.88^{*}$ & & $.89^{*}$ \\
\hline Mi vida es como yo quiero que sea & $.78^{*}$ & $.78^{*}$ & & $.78^{*}$ \\
\hline Me gustaría cambiar muchas cosas en mi vida $(i)$ & $.22 *$ & & $.38^{*}$ & \\
\hline Me gustaría tener otro tipo de vida $(i)$ & $.35^{*}$ & & $1.47^{*}$ & \\
\hline
\end{tabular}

Nota: $*=p<.001 ; i=$ ítem inverso; $A F E=$ Análisis Factorial Exploratorio; $A F C=$ Análisis Factorial Confirmatorio.

Por medio de un $A F C$ se procedió a comparar la solución de un solo factor versus dos factores (uno de ellos compuesto por 5 ítems y el otro con los 2 ítems invertidos). En la Tabla 3 se observan las cargas factoriales de los ítems para ambos factores. Por otra parte, los índices de ajuste del modelo bidimensional tuvieron un incremento importante en comparación a los del modelo de un factor con siete ítems (Tabla 4). Finalmente, la correlación entre ambos factores fue de $.18(p<.01)$. 
Tabla 4. Índices de ajuste para los modelos evaluados de la SLSS.

\begin{tabular}{lcccccc}
\hline Modelo & $\chi^{2}$ & $g l$ & CFI & TLI & RMSEA & WRMR \\
\hline Un factor con 7 ítems & $688.96^{*}$ & 14 & .79 & .68 & $.30($ IC 90\% $=.28-.32)$ & 2.66 \\
Dos factores & $98.34^{*}$ & 13 & .97 & .96 & .11 (IC 90\%=.09-.13) & .91 \\
Un factor con 5 ítems & $48.84^{*}$ & 5 & .98 & .97 & .12 (IC 90\%=.09-.16) & .68 \\
\hline
\end{tabular}

Nota: $*=p<.001 ; \chi^{2}=$ Chi Cuadrado; $g l=$ grados de libertad; CFI = Comparative Fit Index; TLI = Tucker Lewis Index; RMSE $A=$ Root-Mean-Square Error or Approximation; $W R M R=$ Weighted Root Mean Square Residual.

A pesar de que estos resultados son positivos, encontramos que el ítem 7 ("Me gustaría tener otro tipo de vida") obtuvo una carga factorial superior a 1.0. En casos como éste, Joreskog (1999) sugiere revisar la varianza residual del ítem: si ésta es positiva puede aceptarse dicha solución; por el contrario, si es negativa, entonces la solución es teóricamente improcedente. En nuestro caso, la varianza residual del ítem 7 resultó negativa. Como consecuencia de esto, se decidió realizar un $A F C$ con el factor de 5 ítems. En la Tabla 3 se observa que con esta solución, todos los ítems obtienen una carga igual o encima de .50. En cuanto a los índices de ajuste, estos fueron bastante similares a los obtenidos por el modelo de dos factores, pero claramente la solución de un factor es más parsimoniosa y similar a lo reportado en otras investigaciones.

\section{Conclusiones y Discusión}

La presente investigación describe la validación del Student's Life Satisfaction Scale (SLSS) elaborado por Huebner (1991), en una muestra de 1096 escolares chilenos entre 10 y 12 años. El estudio aporta con una herramienta útil para la generación de conocimiento y estrategias de investigación que son necesarias en materia de infancia en América Latina, pues como se ha planteado, existen pocas escalas validadas y poca información en este ámbito para este rango etario, al mismo tiempo que gran preocupación respecto al bienestar de los niños y niñas, particularmente en el grupo de edad específico que fue estudiado en este trabajo. De esta manera se aporta al desarrollo de herramientas de evaluación psicométrica disponibles para la población infantil chilena, relevando así la situación de bienestar subjetivo de niños y niñas entre 10 y 12 años de países en vías de desarrollo, aportando con ello a la evaluación y desarrollo de recursos para establecer comparaciones locales e internacionales.

Complementariamente a lo anterior, instrumentos de este tipo podrían permitir el estudio y aportar al desarrollo de intervenciones en ámbitos relacionados con salud mental infantil. Además, puede ser instrumental útil en la observación de programas y políticas de calidad de vida (o similares) con niños y niñas de esta edad, lo que ayuda de manera importante a la toma de decisiones en políticas públicas y programas de infancia (Castellanos, 2012). Un hallazgo relevante fue que, a diferencia de lo encontrado en un estudio de validación de esta misma escala realizado con adolescentes españoles (Galindez \& Casas, 2010), entre los niños y niñas chileno/as entre 10 y 12 años se encontró una estructura bidimensional en vez de una unidimensional. El primer factor integra ítems que claramente se refieren al bienestar general mientras que el segundo incluye los ítems inversos relacionados con la intención de cambio.

Ante estos resultados, una pregunta que surge naturalmente es respecto de las razones de esta estructura de dos factores. Si bien la literatura señala que en una escala multidimensional, un mínimo de tres ítems debieran cargar en cada factor, algunos autores plantean que también puede considerarse dos ítems por factor, aunque esto debiera ser analizado a la luz de la teoría disponible (Raubenheimer, 2004). Creemos que en este caso pudiera justificarse un segundo factor con sólo dos ítems. Al respecto se podría hipotetizar que la existencia de dos factores se relaciona con la existencia de ítems invertidos, lo que a su vez podría verse afectado por el nivel de comprensión lectora reportada en niños chilenos. En la misma línea, los resultados sugieren que alguna característica idiosincrásica del grupo etario podría significar una dificultad para responder adecuadamente para niños y niñas específicamente de la edad estudiada.

Desde un punto de vista estrictamente metodológico, la presencia de dos factores puede ser explicado debido al sesgo o efecto del método (Conway, 2002). Particularmente en el SLSS los dos ítem invertidos no incluyen una negación explícita, que es una forma común de invertir los ítems, simplemente mediante la negación de la afirmación (Morales, 2006). Lo anterior no obstante no parece un problema en sí mismo, sí tiene un efecto sobre los resultados en los análisis estadísticos. Es necesario aclarar, que acogemos aquí la distinción hecha entre ítems invertidos y en negativo que adoptara Tomás, Sancho, Oliver, Galiana, y Meléndez (2012). Según estos autores los primeros corresponden a la formulación más aconsejada en la construcción de escala, como una forma de evitar la aquiescencia. En tal sentido, debe invertirse el puntaje para interpretar en el sentido correcto la afirmación según la dirección de los demás ítems de la escala. Por otra parte, los ítems en negativo tienen incluida una negación en el anunciado, tales como "no" o "nunca", y que típicamente se desaconseja su uso por la posibilidad que el participante responda de forma equívoca o que implique una doble negación. Los autores demostraron que el efecto de método se produce con ítems invertidos y no con ítems en negativo, efecto que resulta nulo o casi nulo en el segundo tipo. Para comprobarlo usaron diferentes modelos de análisis con escalas de autoestima y bienestar que tenían ítems inversos y en negativo y fueron sometidos a $A F C$ y diversos modelos de ecuaciones estructurales. Los autores señalan que el invertir ítem parece interferir en el examen de la estructura latente de una escala, lo que se anula cuando los ítems están redactados de 
forma positiva o en negativo. Para mayor detalle consultar trabajo de Tomás et al. (2012) y Tomás, Galiana, Hontangas, Oliver, y Sancho (2013).

La evidencia de una estructura bifactorial, donde un factor está constituido por dos ítems invertidos resulta plausible si se considera, además, que en Chile un importante porcentaje de estudiantes tienen dificultades de comprensión lectora, encontrándose por debajo de la media de resultados obtenidos en la prueba PISA por los países OCDE (Agencia Calidad de la Educación, 2014). De manera que esto podría ser entendido como resultado de la dificultad en la comprensión lectora que genera un ítem invertido. Si se tratara de esta situación, es importante reconsiderar dichos ítems ya que no serían pertinentes en un contexto de auto-reporte a esta edad, al menos en condiciones educacionales como la chilena o similares a ésta. Es presumible suponer, en base a la evidencia aportada por Tomás et al. (2012, 2013), más el efecto asociado a la comprensión lectora, que la estructura factorial del SLSS sea unidimensional como lo han presentado la mayoría de las investigaciones (Dew \& Huebner, 1994; Galindez \& Casas, 2010; Huebner, 1991; Marques et al., 2007; Weber et al., 2013).

Otra hipótesis posible es que la existencia de dos factores podría deberse al sentido que los participantes le hayan dado a los dos ítems que cargan en el segundo factor, pues la redacción de éstos difiere del resto de los ítems, en cuanto, para responderlos se requiere que los niños y niñas realicen un examen o cálculo de su vida en un sentido futuro, lo que también constituiría un efecto de método, como el ya señalado. Independientemente de lo anterior, parece de por sí interesante analizar y tratar de comprender la idiosincrasia de los niños y niñas de esta edad, donde pareciera existir una visión de bienestar subjetivo con una lógica diferente a la reportada para los adolescentes o los adultos. Así, se podría suponer que la vivencia o desarrollo de bienestar en determinadas edades ocurre de manera diferente, asumiendo que las fuentes que determinan el bienestar son distintas a diferentes edades (Casas, 2010a).

Esta particularidad de la idiosincrasia de la infancia con respecto al bienestar podría relacionarse con la diferenciación que tendría aspectos del bienestar presente e inmediatos, respecto de aspectos de futuro, que supone un plano más abstracto de reporte de satisfacción con la vida, por lo que podría verse afectado por los procesos de desarrollo cognitivo que tienen lugar en la pubertad y adolescencia. La presencia de dos factores podría relacionarse con el sentido o dimensión de la vida que consideran los ítems que cargan en uno y otro factor. Así para los niños y niñas de estas edades la evaluación de la propia situación actual no es lo mismo

\section{Referencias}

Agencia de Calidad de la Educación (2014). Informe nacional de resultados Chile PISA 2012. Recuperado de htps://s3-us-west2.amazonaws.com/documentos-

web/Estudios+Internacionales/PISA/Informe_Nacional_Resultados_ Chile_PISA_2012.pdf que la situación futura esperada o deseada en una proyección futura. Por tanto, podría pensarse que los niños y niñas de nuestra muestra podrían estar asociando la idea del bienestar con la estabilidad. De este modo, el ítem que alude a "cambiar muchas cosas" podría implicar no necesariamente una insatisfacción con las condiciones actuales de su vida.

Si bien teóricamente puede defenderse la bidimensionalidad de la SLSS, estadísticamente no lo es puesto que uno de los ítems invertidos que cargan en el segundo factor obtuvo una varianza residual negativa. En consecuencia, se tomó la decisión de considerar solo los cinco ítems no invertidos que cargaron en el primer factor. De esta manera, nuestra principal conclusión -que dista de ser concluyente- es que la aplicación de la SLSS en una muestra de participantes entre 10 y 12 años tiende a favorecer la presencia de ítems claramente relacionados con la satisfacción con la vida.

Por último, se hace relevante destacar que en este estudio se confirma y valida la posibilidad de aplicar instrumentos de auto-reporte en niños de estas edades, y si bien se debe seguir explorando al respecto, se destaca que instrumentos de este tipo permiten conocer la perspectiva del niño, lo cual es de suma relevancia hoy y coherente con lo planteado en la Convención Internacional de los Derechos de los Niños y Niñas. Basados en este auto-reporte, es interesante mostrar que los niños no sólo reportan una visión general de su vida, sino también, que pueden referirse a diferentes ámbitos de su satisfacción global con la vida, lo que permite analizar con mayor precisión y fineza los elementos involucrados en su bienestar.

Como limitaciones del estudio, es posible señalar que éste consideró solo población infantil urbana, que habita en las tres ciudades con mayor concentración de población de Chile, dejando fuera criterios importantes como etnia, ruralidad, entre otras variables importantes para la comprensión del bienestar subjetivo infantil. En este sentido, los resultados no pueden ser generalizados para poblaciones diferentes a las consideradas en la muestra de este estudio. En estudios futuros pudiera ser relevante controlar el efecto de método, así como evaluar el efecto diferencial que puede tener en este grupo etário el bienestar subjetivo evaluado con ítems referidos a la situación presente de aquellos que connoten una situación futura. Creemos que estos hallazgos pueden ser valiosos no sólo porque aportan evidencia de validez y fiabilidad de un instrumento, sino también permita a otros investigadores estar atentos al efecto de ítems inversos al evaluar bienestar subjetivo en este grupo etário, particularmente por la posibilidad que ello derive en una modelo bifactorial para la SLSS.

Aguado, L. (2009). Un índice de no consumo de alimentos en la niñez para Colombia. Revista Latinoamericana de Ciencias Sociales, Niñez y Juventud, 7(2), 735-760.

Alfaro, J., Guzmán, J., García, C., Sirlopú, D., Gaudlitz, L., \& Oyanedel, J.C. (2015). Propiedades psicométricas de la Escala Breve Multidimen- 
sional de Satisfacción con la Vida en Estudiantes (BMSLSS) en población infantil chilena (10-12 años). Universitas Psychologica, 14(1), 15-27.

Alfaro, J., Valdenegro, B., \& Oyarzún, D. (2013). Análisis de propiedades psicométricas del índice de bienestar personal en una muestra de adolescentes chilenos. Diversitas: Perspectivas en Psicología, 9(1), 13-27.

Athay, M. M., Kelley, S. D., \& Dew-Reeves, S. E. (2012). Brief multidimensional students' life satisfaction scale-PTPB version (BMSLSS-PTPB): Psychometric properties and relationship with mental health symptom severity over time. Administration and Policy in Mental Health, 39(1-2), 30-40. doi:10.1007/s10488-011-0385-5

Ato, M., López, J., \& Benavente, A. (2013). Un sistema de clasificación de los diseños de investigación en psicología. Anales de Psicología, 29(3),1038-1059. doi:10.6018/analesps.29.3.178511

Beauducel, A., \& Herzberg, P. Y. (2006). On the performance of maximum likelihood versus means and variance adjusted weighted least squares estimation in CFA. Structural Equation Modeling, 13, 186-203.

Ben-Arieh, A. (2008). The child indicators movement: Past, present and future. Child Indicators Research, 1, 3-16. doi:10.1007/s12187-007-9003-1

Bradshaw, J., \& Lau, M. (2010). Child well-being in the Pacific rim. Child Indicators Research, 3(3), 367-383. doi:10.1007/s12187-010-9064-4

Bradshaw, J., \& Richarson, D. (2009). An index of child well-being in Europe. Child Indicators Research, 2(3), 319-351. doi:10.1007/s12187-0099037-7

Campbell, A., Converse, P., \& Rodgers, W. (1976). The quality of American life: Perceptions, evaluations and satisfactions. New York, NY: Russell Sage Foundation.

Casas, F. (2010a). El bienestar personal: Su investigación en la infancia y la adolescencia. Encuentros en Psicología Social, 5(1), 85-101.

Casas, F. (2010b). Indicadores sociales subjetivos y bienestar en la infancia y adolescencia. En UNICEF (Ed.), Propuesta de un sistema de indicadores sobre bienestar infantil en España (pp. 34-51). Madrid: UNICEF España.

Casas, F., Sarriera, J. C., Abs, D., Coenders, G., Alfaro, J., Saforcada, E., \& Tonon, G. (2011). Subjective indicators of personal well-being among adolescents. Performance and results for different scales in Latinlanguage speaking countries: A contribution to the international debate. Child Indicators Research, 5(1), 1-28. doi:10.1007/s12187-011-9119-1

Casas, F., Sarriera, J. C., Alfaro, J., González, M., Malo, S., Bertrán, I., ... Valdenegro, B. (2012). Testing the personal wellbeing index on 12-16 year-old adolescents in 3 different countries with 2 new items. Social Indicators Research, 105(3), 461-482. doi:10.1007/s11205-011-9781-1

Castellanos, R. (2012). El bienestar subjetivo como enfoque e instrumento de la politica pública: Una revisión analítica de la literatura. Revista Chilena de Administración Pública, 19, 133-168.

Chaves, C., Vazquez, C., \& Hervas, G. (2013). Benefit finding and wellbeing in children with life threatening illnesses: An integrative study. Terapia Psicológica, 31(1), 59-68.

CONICYT/FONDECYT (2008). Bioética en Investigación en Ciencias Sociales. Santiago: Ministerio de Educación-Chile.

Conway, J. M. (2002). Method variance and method bias in industrial and organizational psychology. In S. G. Rogelberg (Ed.), Handbook of research methods in industrial and organizational psychology (pp. 344-365). Malden, MS: Blackwell Publishers Inc.

Cuesta, M., Fonseca-Pedrero, E., Vallejo, G., \& Muñiz, J. (2013). Datos perdidos y propiedads psicométricas en los tests de personalidad. Anales de Psicología, 29(1), 285-292.

Cummins, R. A., Eckersley, R., Van Pallant, J., Van Vugt, J., \& Misajon, R. (2003). Developing a national index of subjective well-being: The Australian unity well-being index. Social Indicators Research, 64,159-190. doi:10.1023/A:1024704320683

Dew, T., \& Huebner, E.S. (1994). Adolescents' perceived quality of life: An exploratory investigation. Journal of School Psychology, 33, 185-199.

Diener, E. (1984). Subjective well-being. Psychological Bulletin, 95(3), 542-575.

Diener, E. (2006). Guidelines for national indicators of subjective wellbeing and ill-being. Applied Research in Quality of Life, 1(2), 151-157. doi:10.1007/s11482-006-9007-x

Diener, E., \& Suh, E. (1997). Measuring quality of live: Economic, social, and subjetive indicators. Social Indicators Research, 40, 189-216. doi:10.1023/A:1006859511756
Domínguez, S. (2012). Propuesta para el cálculo del alfa ordinal y theta de Armor. Revista de Inmvestigación en Psicología, 15(1), 213-217.

Domínguez, S. (2014). ¿Matrices Policóricas/Tetracóricas o matrices de Pearson? Un estudio metodológico. Revista Argentina de Ciencias del Comportamiento, 6(1), 39-48

Elosua, P., \& Zumbo, B. (2008). Coeficientes de fiabilidad para escalas de respuesta categórica ordenada. Psicothema, 20(4), 896-901.

Ferrando, P., \& Lorenzo-Seva, U. (1994). Recuperación de la solución factorial a partir de variables dicotomizadas. Psicothema, 6(3), 483-491.

Ferrando, P., \& Lorenzo-Seva (2014). El análisis factorial exploratorio de los ítems: algunas consideraciones adicionales. Anales de Psicología, 30(3), 1170-1175

Figuer, C., Casas, F., Buxarrais, M., González, M., Tey, A., Noguera, E., \& Rodriguez, J. (2004). Los valores y su influencia en la satisfacción vital de los adolescentes entre los 12 y los 16 años: estudio de algunos correlatos. Apuntes de Psicología, 22(1), 3-23.

Freiberg, A., Stover, J., de la Iglesia, G., \& Fernández, M. (2013). Correlaciones policóricas y tetracóricas en estudios factoriales exploratorios y confirmatorios. Ciencias Psicológicas, 7(2),151-164.

Gadermann, A. M., Guhn, M., \& Zumbo, B. (2012). Estimating ordinal reliability for Likert-type and ordinal item response data: A conceptual, empirical, and practical guide. Practical Assessment, Research \& Evaluation, 17(3), 1-13.

Gadermann, A. M., Schonert-Reichl, K. A., \& Zumbo, B. (2010). Investigating validity evidence of the satisfaction with life scale adapted for children. Social Indicators Research, 96, 229-247. doi:10.1007/s11205009-9474-1

Galindez, E., \& Casas, F. (2010). Adaptación y validación de la students' life satisfaction scale (SLSS) con adolescentes. Estudios de Psicología, 31(1), $79-87$.

Gallardo, I., \& Moyano, E. (2012). Análisis psicométrico de las escalas Ryff (versión española) en una muestra de adolescentes chilenos. Universitas Psychologica, 11(3), 931-939.

Hu, L., \& Bentler, P. (1999). Cutoff criteria for fit indexes in covariance structure analysis: conventional criteria versus new alternatives. Structural Equation Modeling, 6, 1-55.

Huebner, E. S. (1991). Initial development of the student's life satisfaction scale. School Psychology International, 12(3), 231-240. doi:10.1177/0143034391123010

Huebner, E. S. (2004). Research on assessment of life satisfaction of children and adolescents. Social Indicators Research, 66(1/2), 3-33. doi:10.1023/B:SOCI.0000007497.57754.e3

Huebner, E. S., \& Gilman, R. (2002). An introduction to the multidimensional students' life satisfaction scale. Social Indicators Research, 60, 115-122. doi:10.1023/A:1021252812882

Huebner, E. S., Suldo, S. M., \& Valois, F. (2003). Psychometric properties of two brief measures of Children's Life Satisfaction Scale (SLSS) and the Brief Multidimensional Student's Life Satisfaction Scale (BMSLSS). Paper For Indicators of Positive Development Conference, Washington, EEUU. Disponible content/uploads/2013/05/Child_Trends20030312 PD PDConfHSVP.pdf

Huebner, E. S., Suldo, S. M., Valois, R. F., \& Drane, J. W. (2006). The brief multidimensional students' life satisfaction scale: Sex, race, and grade effects for applications with middle school students. Applied Research in Quality of Life, 1(2), 211-216. doi:10.1007/s11482-006-9016-9

Jiang, X., Huebner, E. S., \& Hills, K. (2013). Parent attachment and early adolescents' life satisfaction: The mediating effect of hope. Psychology in the Schools, 50(4), 340-352. doi:10.1002/pits.21680

Joreskog, K. G. (1999). How large can a standardized coefficient be? Unplublished report. SSI Central, Inc.

Kline, R. B. (2011). Principles and practice of structural equation modeling (3rd ed.). New York, NY: The Guilford Press.

Lau, A. L. D., Cummins, R. A., \& McPherson, W. (2005). An investigation into the cross-cultural equivalence of the personal wellbeing index. Social Indicators Research, 72, 403-432. doi:10.1007/s11205-004-0561-z

Lei, P. W. (2009). Evaluating estimation methods for ordinal data in structural equation modeling. Quality and Quantity, 43, 495-507. 
Llorent-Segura, S., Ferreres-Traver, A., Hernández-Baeza, A., \& TomásMarco, I. (2014). El análisis factorial exploratorio de los ítems: una guía práctica, revisada y actualizada. Anales de Psicología, 30(3), 1151-1169

Lorenzo-Seva, U., \& Ferrando, P. J. (2006). FACTOR: A computer program to fit the exploratory factor analysis model. Behavioral Research Methods, Instruments and Computers, 38(1), 88-91.

Mardia, K. (1970). Measures of multivarinte skewness and kurtosis with applications. Biometrika, 57, 519-530.

Mardia, K. (1974). Applications of some measures of multivariate skewness and kurtosis in testing normality and robustness studies. Sankbya, 36, 115-128.

Marques, S. C., Pais-Ribeiro, J. L., \& Lopez, S. J. (2007). Validation of a Portuguese version of the students' life satisfaction scale. Applied Research in Quality of Life, 2(2), 83-94. doi:10.1007/s11482-007-9031-5

McKnight, C. G., Huebner, E. S., \& Suldo, S. (2002). Relationships among stressful life events, temperament, problem behavior, and global life satisfaction in adolescents. Psychology in the Schools, 39(6), 677-687. doi:10.1002/pits.10062

Montero, I., \& León. O. (2007). A guide for naming research studies in Psychology. International Journal of Clinical ans Health Psychology, 7(3),847862.

Morales, P. (2006). Medición de actitudes en psicología y educación (3ra Edición Revisada). Madrid: Universidad Pontificia de Comillas de Madrid.

Moyano, E., Flores, E., \& Soromaa, H. (2011). Fiabilidad y validez de constructo del test MUNSH para medir felicidad, en población de adultos mayores chilenos. Universitas Psychologica, 10(2), 567-580.

Muñiz, J., Elousa, P., \& Hambleton, R. (2013). Directrices para la traducción y adaptación de los test: segunda edición. Psicothema, 25(2), 151-157.

Muthén, L.K. \& Muthén, B.O. (2012). Mplus user's guide. (7th ed.). Los Angeles, CA: Muthén \& Muthén.

Muthén, B., du Toit, S. H. C., \& Spisic, D. (1997). Robust inference using weighted least squares and quadratic estimating equations in latent variable modeling with categorical and continuous outcomes. Unpublished technical report. Artículo recuperado de: http://www.statmodel.com/bmuthen/articles/Article_075.pdf

Olson, U. (1979). On the robustness of factor analysis against crude classification of the observations. Multivariate Behavioral Research, 14, 485-500.

Pavot, W., \& Diener, E. (1993). Review of the satisfaction with life scale. Psychological Assessment, 5, 164-172. doi:10.1037/1040-3590.5.2.164

Paxton, R. J., Valois, R. F., Huebner, E. S., \& Drane, J. W. (2006). Opportunity for adult bonding/meaningful neighborhood roles and life-satisfaction among USA middle school students. Social Indicators Research, 79(2), 291-312. doi:10.1007/s11205-005-4129-3

Petito, F., \& Cummins, R. A. (2000). Quality of life in adolescence: The role of perceived control, parenting style, and social support. Behaviour Change, 17(3), 196-207. doi:10.1375/bech.17.3.196

Pollard, E. L., \& Lee, P. D. (2003). Child wellbeing: A systematic review of the literature. Social Indicators Research, 61, 59-78. doi:10.1023/A:1021284215801

Puente, R., \& Cavazos, J. (2013). Personality factors, affect, and autonomy support as predictors of life satisfaction. Universitas Psychologica, 12(1), 41-53.

Raubenheimer, J. (2004). An item selection procedure to maximise scale reliability and validity. SA Journal of Industrial Psychology, 30(4), 59-64. doi: 10.4102/ sajip.v30i4.168

Seligson, J. L., Huebner, E. S., \& Valois, R. F. (2003). Preliminary validation of the brief multidimensional students' life satisfaction scale (BMSLSS). Social Indicators Research, 61, 121-145. doi:10.1023/A:1021326822957
Seligson, J. L., Huebner, E. S., \& Valois, R. F. (2005). An investigation of a brief life satisfaction scale with elementary school children. Social Indicators Research, 73(3), 355-374. doi:10.1007/s11205-004-2011-3

Siyez, D. M., \& Kaya, A. (2008). Validity and reliability of the brief multidimensional students' life satisfaction scale with Turkish children. Journal of Psychoeducational Assessment, 26(2), 139-147. doi:10.1177/0734282907307802

Tiliouine, H., Cummins, R., \& Davern, M. (2006). Measuring wellbeing in developing countries: The case of Algeria. Social Indicators Research, 75 , 1-30. doi:10.1007/s11205-004-2012-2

Tomás, J. M., Galiana, L., Hontangas, P., Oliver, A., \& Sancho, P. (2013). Evidencia acumulada sobre los efectos de método asociado a ítems invertidos. Psicológica, 34, 365-381.

Tomás, J.M., Sancho, P.,Oliver, A., Galiana, L., \& Meléndez, J.C. (2012). Efectos de método asociados a ítems invertidos vs. ítems en negativo. Revista Mexicana de Psicología, 29(2), 105-115.

Tomyn, A. J., \& Cummins, R. A. (2011). The subjective wellbeing of highschool students: Validating the personal wellbeing index-schoo children. Social Indicators Research, 101(3), 405-418. doi:10.1007/s11205010-9668-6

Ullman, J. B. (2006). Structural equation modeling. In B. G. Tabachnick y L. S. Fidell (Eds.), Using multivariate statistics (5th ed., pp. 653-771) Boston, MA: Allyn \& Bacon.

Valois, R. F., Zullig, K. J., Huebner, E. S., \& Drane, J. W. (2003). Dieting behaviors, weight perceptions, and life satisfaction among public high school adolescents. Eating Disorders, 11(4), 271-288. doi:10.1080/10640260390242506

Valois, R. F., Zullig, K. J., Huebner, E. S., \& Drane, J. W. (2004a). Life satisfaction and suicide among high school adolescents. Social Indicators Research, 66, 81-105. doi:10.1023/B:SOCI.0000007499.19430.2f

Valois, R. F., Zullig, K. J., Huebner, E. S., \& Drane, J. W. (2004b). Physical activity behaviors and perceived life satisfaction among public high school adolescents. Journal School of Health, 74(2), 59-65. doi:10.1111/j.1746-1561.2004.tb04201.x

Veenhoven, R. (1994). El estudio de la satisfacción con la vida. Intervención Psicosocial, 3(9), 87-116.

Veenhoven, R. (2002). Why social policy needs subjective indicators. Social Indicators Research, 58(1), 33-46. doi:10.1007/0-306-47513-8_3

Vera-Villarroel, P., Urzúa, A., Pavez, P., Celis-Atenas, K., \& Silva, J. (2012). Evaluation of subjective well-being: Analysis of the satisfaction with life scale in Chilean population. Universitas Psychologica, 11(3), 719-727.

Webb, D. (2009). Subjective wellbeing on the Tibetan plateau: An exploratory investigation. Journal of Happiness Studies, 10(6), 753-768. doi:10.1007/s10902-008-9120-7

Weber, M., Ruch, W., \& Huebner, E. S. (2013). Adaptation and initial validation of the German version of the students' life satisfaction scale (German SLSS). European Journal of Psychological Assessment, 29(2), 105 112. doi:10.1027/1015-5759/a000133

West, S. G., Taylor, A. B, \& Wu, W. (2012). Model fit and model selection in structural equation modeling. En R. H Hoyle (Ed.), Handbook of structural equation modeling (pp. 209-231). New York, NY: The Guilford Press.

Yiengprugsawan, V., Seubsman, S., Khamman, S., \& Lim, L. L. (2010). Personal wellbeing index in a national cohort of 87,134 Thai adults. Social Indicators Research, 98, 201-215. doi:10.1007/s11205-009-9542-6

(Articulo recibido: 15-01-2015; revisado: 16-05-2015; aceptado: 31-07-2015) 